\title{
Towards Stealthy Malware Detection 1
}

\author{
Salvatore J. Stolfo, Ke Wang, Wei-Jen Li \\ Department of Computer Science
}

Columbia University

\begin{abstract}
Malcode can be easily hidden in document files and go undetected by standard technology. We demonstrate this opportunity of stealthy malcode insertion in several experiments using a standard COTS Anti-Virus (AV) scanner. Furthermore, in the case of zero-day malicious exploit code, signature-based AV scanners would fail to detect such malcode even if the scanner knew where to look. We propose the use of statistical binary content analysis of files in order to detect suspicious anomalous file segments that may suggest insertion of malcode. Experiments are performed to determine whether the approach of n-gram analysis may provide useful evidence of a tainted file that would subsequently be subjected to further scrutiny. We further perform tests to determine whether known malcode can be easily distinguished from otherwise "normal" Windows executables, and whether self-encrypted files may be easy to spot. Our goal is to develop an efficient means by static content analysis of detecting suspect infected files. This approach may have value for scanning a large store of collected information, such as a database of shared documents. The preliminary experiments suggest the problem is quite hard requiring new research to detect stealthy malcode.
\end{abstract}

\footnotetext{
${ }^{1}$ This work was partially supported by a grant from ARDA under a contract with
} Batelle, Pacific Northwest Labs. 


\section{Introduction}

Attackers have used a variety of ways of embedding malicious code in otherwise normal appearing files to infect systems. Viruses that attach themselves to system files, or normal appearing media files, are nothing new. State-of-the-art COTS products scan and apply signature analysis to detect these known malware. For various performance optimization reasons, however, COTS Anti-Virus (AV) scanners may not perform a deep scan of all files in order to detect known malcode that may have been embedded in an arbitrary file location. Other means of stealth to avoid detection are well known. Various self-encryption or code obfuscation techniques may be used to avoid detection simply making the content of malcode unavailable for inspection by an $\mathrm{AV}$ scanner. In the case of new zero day malicious exploit code, signature-based AV scanners would fail to detect such malcode even if the scanner had access to the content and knew where to look.

In this chapter we explore the use of statistical content analysis of files in order to detect anomalous file segments that may suggest infection by malcode. Our goal is to develop an efficient means of detecting suspect infected files for application to scanning a large store of collected information, such as a database of content in a file sharing network. The work reported in this chapter is preliminary. Our ongoing studies have uncovered a number of other techniques that are under development and evaluation. Here we present background summary on our work on Fileprints, followed by several experiments applying the method to malcode detection.

The threat model needs to be clarified in this work. We do not consider the methods by which stealthy malcode embedded in tainted files may be automatically launched and executed. One may posit that detecting a tainted file may be easy simply by opening the file and detecting whether the application issues a fault. This might be the case if the malcode was embedded in such a way as to damage the expected file format causing the application to fault. As we show in section 2, one can embed malcode without creating such a fault when opening a tainted file. In this work, we focus specifically on static analysis techniques to determine whether or not we may be able to identify a tainted file. The approach we propose is to use generic statistical feature analysis of binary content irrespective of the type of file used to transport the malcode into a protected environment.

Files typically follow naming conventions that use standard extensions describing its type or the applications used to open and process 
the file. However, although a file may be named Paper.doc ${ }^{2}$, it may not be a legitimate Word document file unless it is successfully opened and displayed by Microsoft Word, or parsed and checked by tools, such as the Unix file command, if such tools exist for the file type in question. We proposed a method to analyze the contents of exemplar files using statistical modeling techniques. In particular, we apply n-gram analysis to the binary content of a set of exemplar "training" files and produce normalized n-gram distributions representing all files of a specific type. Our aim is to determine the validity of files claiming to be of a certain type (even though the header may indicate a certain file type, the actual content may not be what is claimed) or to determine the type of an unnamed file object.

The conjecture is that we may model different types of files to produce a model of what all files of that type should look like. Any significant deviation from this model may indicate the file is infected with embedded malcode. Suspect files identified using this technique may then be more deeply analyzed using a variety of techniques under investigation by other researchers (e.g., $[9,16,18]$.)

In our prior work $[11,19,20]$, we demonstrated an efficient statistical n-gram method to analyze the binary contents of network packets and files. This work followed our earlier work on applying machine learning techniques applied to binary content to detect malicious email attachments [15]. The method trains n-gram models from a collection of input data, and uses these models to test whether other data is similar to the training data, or sufficiently different to be deemed an anomaly. The method allows for each file type to be represented by a compact representation of statistical n-gram models. Using this technique, we can successfully classify files into different types, or validate the declared type of a file, according to their content, instead of using the file extension only or searching for embedded "magic numbers" [11] (that may be spoofed).

We do not presume to replace other detection techniques, but rather to augment approaches with perhaps new and useful evidence to detect suspicious files. Under severe time constraints, such as real-time testing of network file shares, or inspection of large amounts of newly acquired media, the technique may be useful in prioritizing files that are subjected to a deeper analysis for early detection of malcode infection.

\footnotetext{
${ }^{2}$ For our purposes here, we refer to .DOC as Microsoft Word documents, although other applications use the .DOC extension such as Adobe Framemaker, Interleaf Document Format, and Palm Pilot format, to name a few.
} 
In the next section, we describe some simple experiments of inserting malware into normal files and how well a commercial AV scanner performed in detecting these infected files. Amazingly, in several cases the tainted files were opened without problem by the associated application. Section 3 summarizes our work on fileprints using 1-gram distributions for pedagogical reasons. The same principles apply to higher order grams. We present several experiments using these techniques to detected infected files. Our concluding remarks in section 4 identify several areas of new work to extend the preliminary ideas explored in this paper.

\section{Deceiving anti-virus software}

Malware may be easily transmitted among machines as (P2P) network shares. One possible stealthy way to infect a machine is by embedding the malicious payload into files that appear normal and that can be opened without incident. A later penetration by an attacker or an embedded Trojan may search for these files on disk to extract the embedded payload for execution or assembly with other malcode. Or an unsuspecting user may be tricked into launching the embedded malcode in some crafty way. In the latter case, malcode placed at the head of a PDF file can be directly executed to launch the malicious software. Social engineering can be employed to do so. One would presume that an AV scanner can check and detect such infected file shares if they are infected with known malcode for which a signature is available. The question is whether a commercial AV scanner can do so. Will the scanning and pattern-matching techniques capture such embeddings successfully? An intuitive answer would be "yes". We show that is not so in all cases.

We conducted the following experiments. First we collected a set of malware [22], and each of them was tested to verify they can be detected by a COTS anti-virus system ${ }^{3}$. We concatenate each of them to normal PDF files, both at the head and tail of the file. Then we manually test whether the COTS AV can still detect each of them, and whether Acrobat can open the PDF file without error. These tests were performed

${ }^{3}$ This work does not intend to evaluate nor denigrate any particular COTS product. We chose a widely used AV scanner that was fully updated at the time the tests were performed. We prefer not to reveal which particular COTS AV scanner was used. It is not germane to the research reported in this paper. 
on a Windows platform. The results are summarized in table 1 . The COTS anti-virus system has surprisingly low detection rate on these infected files with embedded malware, especially when malware is attached at the tail. For those that were undetected, quite a few can still be successfully opened by Acrobat appearing exactly as the untouched original file. Thus, the malcode can easily reside inside a PDF file without being noticed at all. An example of the manipulated PDF file is displayed in figure 1. The apparent reason Adobe Acrobat Reader (version 7.0) opens infected files with no trouble is that it scans the head of a file looking for the PDF "magic numbers" signaling the beginning header meta-data necessary to interpret the rest of the binary content. Thus, the portions passed over by the reader while searching for its header data provides a convenient place to hide malcode.

Table 1. COTS AV detection rate and Acrobat behavior on embedded malcode.

\begin{tabular}{|c|c|c|c|c|}
\hline \multirow{2}{*}{$\begin{array}{c}\text { Total } \\
\text { virus/worm }\end{array}$} & \multicolumn{2}{|c|}{ Virus at the head of PDF } & \multicolumn{2}{c|}{ Virus at the tail of PDF } \\
\cline { 2 - 5 } detect & $\begin{array}{c}\text { Acrobat can } \\
\text { open }\end{array}$ & $\begin{array}{c}\text { AV can } \\
\text { detect }\end{array}$ & $\begin{array}{c}\text { Acrobat can } \\
\text { open }\end{array}$ \\
\hline 223 & $162(72.6 \%)$ & $4 /$ not detected & $43(19.3 \%)$ & $\begin{array}{c}17 / \text { not } \\
\text { detected }\end{array}$ \\
\hline
\end{tabular}

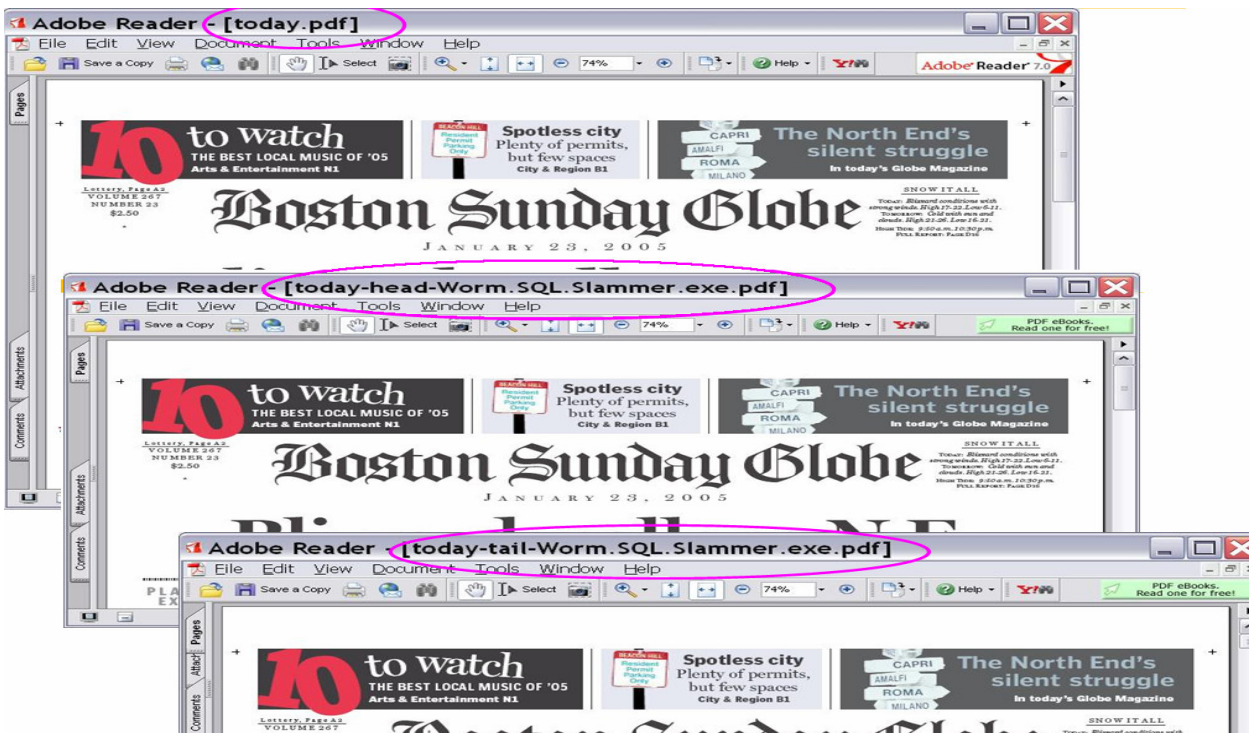

Fig. 1. Screenshot of original and malware embedded PDF file 
We also performed another experiment by inserting the malware into some random position in the middle of the PDF file. But since PDF has its own encoding and such blind insertion can easily break the encoding, generally this is easily noticed by the Acrobat Reader when opening the file. This was the case and hence malware simply appended to the head/tail is obviously easier without causing any errors by the reader. We repeated this experiment on DOC files using some selected malwares, and got a similar result. The following table provides the detailed results of several malware insertion experiments using well known malware. Only CRII can be reliably detected no matter where it is inserted, while Slammer and Sasser were missed.

Table 2. Detailed example of insertion using several well-known malware

\begin{tabular}{|c|c|c|c|}
\hline \multicolumn{4}{|c|}{ Slammer } \\
\hline & Virus at head & In the middle & At tail \\
\hline PDF file & $\begin{array}{l}\text { Not detect/open } \\
\text { fine }\end{array}$ & $\begin{array}{c}\text { Not detect/open } \\
\text { error }\end{array}$ & $\begin{array}{l}\text { Not detect/open } \\
\text { fine }\end{array}$ \\
\hline DOC file & $\begin{array}{l}\text { Not detect/open } \\
\text { error }\end{array}$ & $\begin{array}{c}\text { Not detect/open } \\
\text { error }\end{array}$ & $\begin{array}{c}\text { Not detect/open } \\
\text { fine }\end{array}$ \\
\hline \multicolumn{4}{|c|}{ CodeRed II } \\
\hline \multicolumn{4}{|c|}{ Can be detected anywhere } \\
\hline \multicolumn{4}{|c|}{ Sasser } \\
\hline & Virus at head & In the middle & At tail \\
\hline PDF file & Can detect & $\begin{array}{c}\text { Not detect/open } \\
\text { error }\end{array}$ & $\begin{array}{c}\text { Not detect/open } \\
\text { error }\end{array}$ \\
\hline DOC file & Can detect & $\begin{array}{c}\text { Not detect/open } \\
\text { error }\end{array}$ & $\begin{array}{c}\text { Not detect/open } \\
\text { fine }\end{array}$ \\
\hline
\end{tabular}

Another experiment focused on Windows executables, like WINWORD.EXE. After analyzing the byte value distributions of executables, we noticed that byte value 0 dominated all others. Application executables are stored on disk using a standard block alignment strategy of padding of executables (falling at addresses $n * 4096$ ) for fast disk loading. These zero'ed portions of application files provide ample opportunity to insert hidden malcode. Instead of concatenating malcode, in this case we insert the malcode in a continuous block of 0's long enough to hold the 
whole malcode and store the file back on disk. Again, we tested whether a COTS AV scanner would detect these poisoned applications. It did not. We performed this experiment by replacing the padded segments of WINWORD.EXE, from byte positions 2079784 to 2079848. Figure 2 shows two versions of the application, the normal executable and the other infected with malcode, and both were able to open DOC files with no trouble.

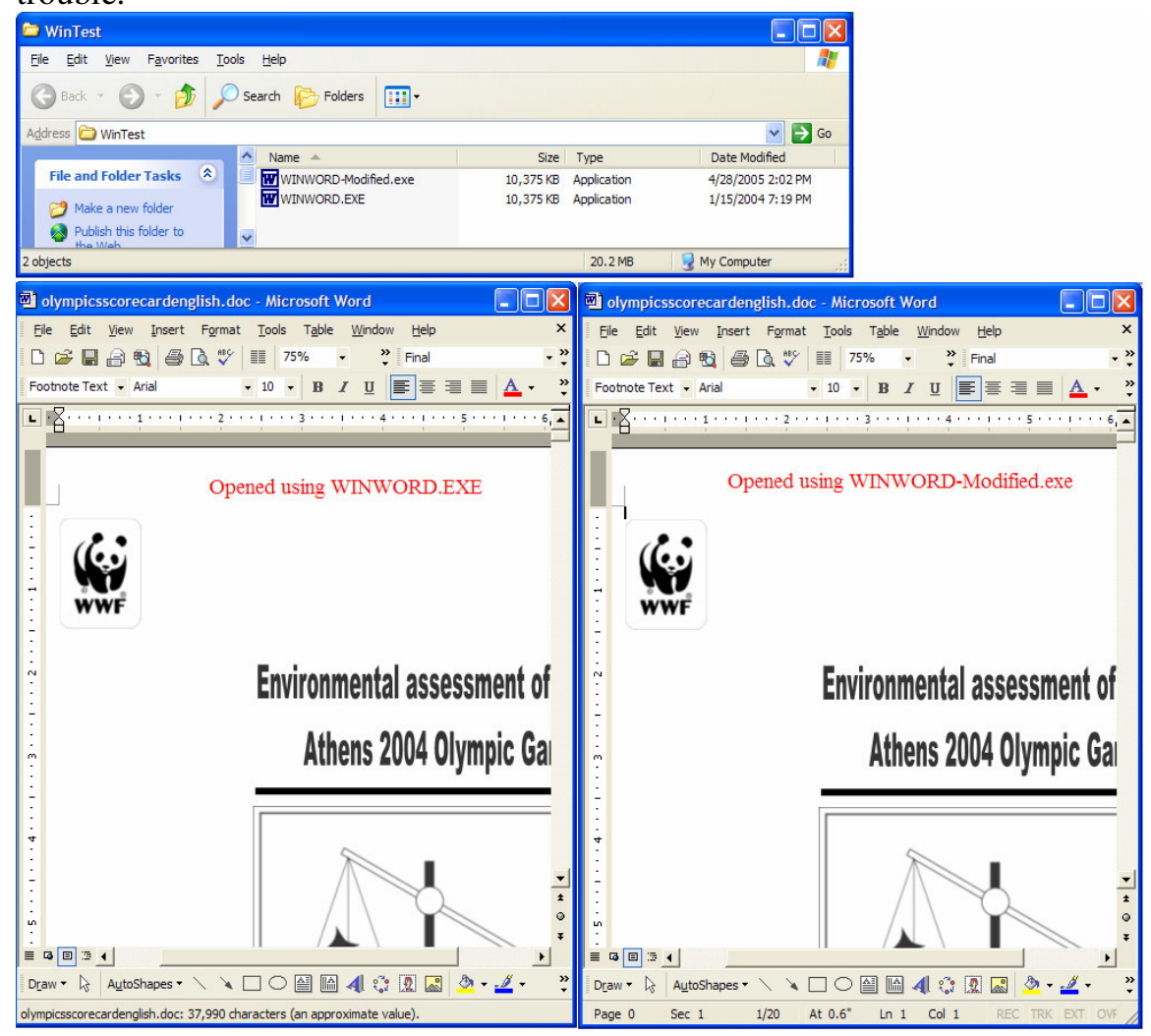

Fig. 2. Opening of a normal DOC file using the original WINWORD.EXE (left) and the infected one WINWORD-Modified.EXE (right). 


\section{3. $\mathrm{N}$-gram experiments on files}

Here we introduce the modeling and testing techniques and present the results of applying these techniques to detect tainted malware-embedded files from normal files of the same type.

\subsection{Fileprints - n-gram distributions of file content}

An $n$-gram [4] is a subsequence of $n$ consecutive tokens in a stream of tokens. N-gram analysis has been applied in many tasks, and is well understood and efficient to implement. By converting a string of data to a feature vector of n-grams, one can map and embed the data in a vector space to efficiently compare two or more streams of data. Alternatively, one may compare the distributions of n-grams contained in a set of data to determine how consistent some new data may be with the set of data in question. In our work to date, we experimented with both 1-gram and 2gram analysis of ASCII byte values. The sequence of binary content is analyzed, and the frequency and variance of each gram is computed. Thus, in the case of 1-grams, two 256-element vectors (histograms) are computed. This is a highly compact and efficient representation, but it may not have sufficient resolution to represent a class of file types. Nevertheless, we test this conjecture by starting with 1-grams. The following plot shows that different file types do indeed have significant distinct 1-gram patterns. Thus, different file types can be reasonably well classified using this technique (see [11]).
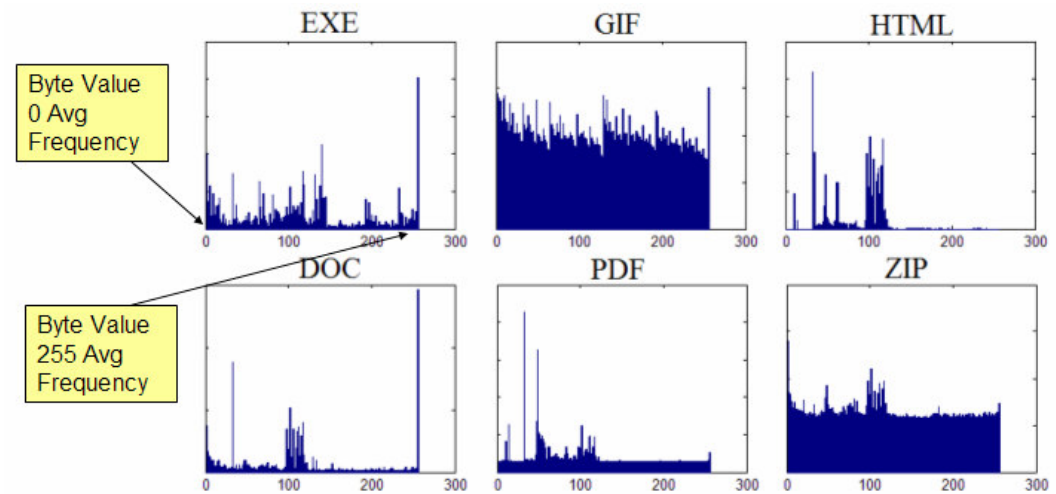

Fig. 3. 1-gram distribution for different file types. 
Once a set of models are computed from a set of normal files, a test file is measured to determine how closely its content conforms to the normal models. This is accomplished by computing the Mahalanobis distance [20] between the test file in question and the normal (centroid) models previously computed. The score produced is a distance measure; a distance threshold is then used to determine whether to declare the file normal or not.

\subsection{Truncation and multiple centroids}

Truncation simply means we model only a fixed portion of a file when computing a byte distribution. That portion may be a fixed prefix, say the first 1000 bytes, or a fixed portion of the tail of a file, as well as perhaps a middle portion. This has several advantages. First, for most files, it can be assumed that the most relevant part of the file, as far as its particular type is concerned, is located early in the file to allow quick loading of metadata by the handler program that processes the file type. Second, viruses often have their malicious code at the very beginning of a file. Hence, viruses may be more readily detected from this portion of the file. However, viruses indeed may also be appended to the end of a file, hence truncation may also be applied to the tail of a file to determine whether a file varies substantially from the expected distribution of that file type. The last, truncation dramatically reduces the computing time for model building and file testing.

On the other hand, files with the same extension do not always have a distribution similar enough to be represented by a single model. For example, EXE files might be totally different when created for different purpose, such as system files, games, or media handlers. Thus, an alternative strategy for representing files of a particular type is to compute "multiple models". We do this via a clustering strategy. Rather than computing a single model $M_{A}$ for files of type A, we compute a set of models $M^{k}{ }_{A}, k>1$. The multiple model strategy requires a different test methodology, however. During testing, a test file is measured against all centroids to determine if it matches at least one of the centroids. The set of such centroids is considered a composite fileprint for the entire class. The multiple model technique creates more accurate models, and separates foreign files from the normal files of a particular type in more precise manner. The multiple models are computed by the $K$-Means algorithm under Manhattan Distance as the similarity metric. The result is a set of $K$ centroid models, $M_{A}^{k}$ which are later used in testing files for various purposes. 


\subsection{Data sets}

To test the effectiveness of the n-gram analysis on files, we conducted several experiments to determine whether it can correctly classify files and whether it can detect malcode.

The test files used in the experiments include 140 PDF files. The malicious files used for embedding were collected from emails, internet sources [22] and some target honeypot machines setup for this purpose in our lab. The PDF files were collected from the internet using a general search on Google. In this way, they can be considered randomly chosen as an unbiased sample. These tests are preliminary; considerable more effort is needed to compose a proper set of training and test data to ensure the files in question represent a true sample of interest. Here we collected documents from an open source and have no means to accurately characterize whether this sample is truly representative of a collection of interest. Nevertheless, this experiment provides some evidence of whether the proposed techniques show promise or not.

\subsection{Detecting malware embedded files}

First we revisit our malcode embedding experiment. We've seen that the COTS AV system we used can easily miss the malcode hidden inside normal appearing files. Here we apply the 1-gram analysis and see how well it may be able to detect the malicious code sequences. 100 of the 140 PDF files were used to build head and tail 1-gram models. Then we tested the remaining 40 normal PDF files and hundreds of malware-embedded files against the model. Since we know ground truth, we measure the detection rate exactly when the false positive rate is zero, i.e., no normal PDF files been misclassified as malware-infected. The result is displayed in table 3 , which is much higher than the COTS anti-virus software detection rate, which for these files is effectively zero. Notice that the total number of malware-embedded files is different for different truncation sizes. That is because the malware used in this study differ in size and we only consider the problem of classifying a pure malcode block fully embedded in a portion of the PDF file. We consider a concatenated PDF file as a test candidate only if the malcode size is equal to or greater than the truncation size used for modeling. 
Table 3. Detection rate using truncated head and tail modeling

\begin{tabular}{|c|c|c|c|}
\hline \multicolumn{4}{|c|}{ Models head N bytes } \\
\hline \multirow{2}{*}{ Detect } & 1000 bytes & 500 bytes & 200 bytes \\
\cline { 2 - 4 } & $49 / 56(87.5 \%)$ & $314 / 347(90.5 \%)$ & $477 / 505(94.5 \%)$ \\
\hline \multicolumn{4}{|c|}{ Models tail N bytes } \\
\hline \multirow{2}{*}{ Detect } & 1000 bytes & 500 bytes & 200 bytes \\
\cline { 2 - 4 } & $42 / 56(75 \%)$ & $278 / 347(80.1 \%)$ & $364 / 505(72.1 \%)$ \\
\hline
\end{tabular}

It may be the case that it is easier to detect the malcode if it is concatenated at the head or tail of a file, since different file types usually have their own standard header information and ending encoding. Malcode may be significantly different from these standardized encodings. However, we test whether malware can effectively be hidden in some middle portion of a file (presuming that the file would still possibly be opened correctly). A reasonable assumption about such insertion is that the malware is inserted as a continuous whole block. So we apply the n-gram detection method to each block of a file's binary content and test whether the model can distinguish PDF blocks from malware blocks. If so, then we can detect the malcode hidden inside PDF files.

We compute byte distribution models using $\mathrm{N}$ consecutive byte blocks from 100 PDF files, then test the blocks of the malware and another 40 PDF files against the model, using Mahalanobis distance. Figure 3 shows the distance of the malware blocks and PDF blocks to the normal model, using $\mathrm{N}=500$ byte blocks and $\mathrm{N}=1000$ byte blocks, respectively. In the plot we display the distance of the malcode blocks on the left side of the separating line and the normal PDF on the right. As the plots show, there is a large overlap between malcode and PDF blocks. The poor results indicate that malware blocks cannot be easily distinguished from normal PDF file blocks using 1-gram distributions. 

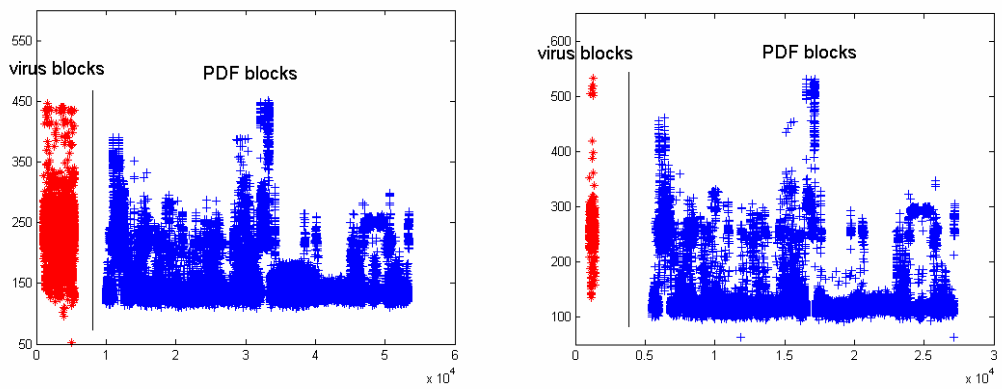

Fig. 4. The Mahalanobis distance of the normal PDF and malware blocks to the trained PDF block model. The left is 500-byte block and the right plot is 1000byte block.

In order to understand why the block-based detection using 1grams does not work well, we plot the byte distribution of each block of a normal PDF file and the Sasser worm code. The first 9 blocks of the PDF file and the first 6 blocks of Sasser are displayed in the following plots. These plots clearly show that different blocks inside a PDF file differ much in their byte distribution, and we cannot determine an absolute difference of the malcode blocks from PDF blocks. Therefore, it appears that a 1-gram statistical content analysis might not have sufficient resolution for malware block detection. Either higher order grams (perhaps 2 -grams or 3-grams) may suffice, or we may need more syntactic information about the file formats to adequately distinguish malcode embedded in PDF files. A search for better statistical features is part of our ongoing research. 

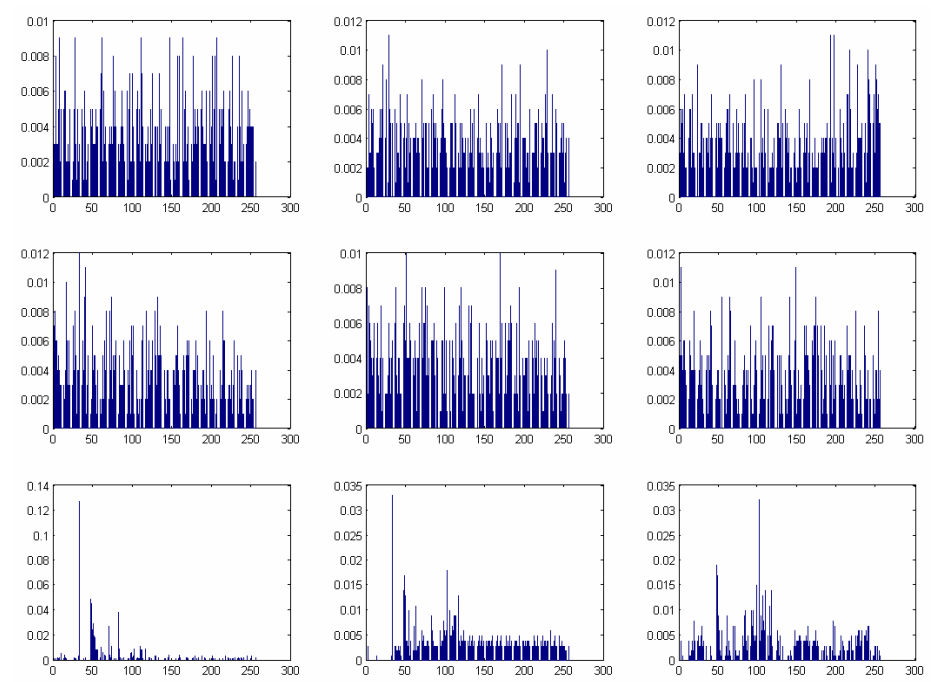

(a) PDF file
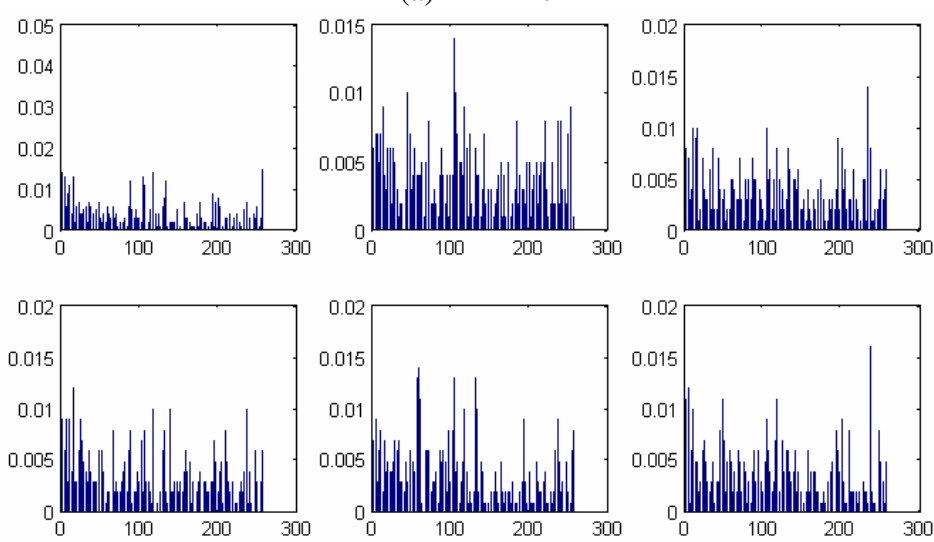

(b) Slammer worm

Fig. 5. Byte value distributions of blocks of the PDF file and Sasser worm.

\subsection{Classifying normal executables and viruses}

In this experiment, we use a collection of malcode executables gathered from other external sources, and compare the 1-gram and 2-gram distributions of these to the corresponding distributions of "normal" Windows executables to determine whether viruses exhibit any clear separating characteristics. We conjecture that the Windows executables are 
generated by programming environments and compilers that may create standard "headers" different from those used by virus writers who deliver their viruses via email or file shares.

We apply three modeling methods to these experiments, which are one-centroid, multi-centroids and exemplar files as centroids. The one centroid method trains one single model for each class (or type) of file. We build $\mathrm{n}$ models $M_{1}, M_{2}, \ldots, M_{n}$, from $n$ different file types. Then, we compute the distance of the test file $F$ to each model, and $F$ is classified to the model with the closest distance.

Alternatively, the multi-centroids method, we build $k$ models $M_{1}^{t}$, $M_{2}^{t}, \ldots, M_{k}^{t}$ using $k$-means algorithm for each file type $t$ as described in section 3.2. There are $k^{*} T$ models in total, where $T$ is the number of file types. $k$ is set to 10 in this test. The test strategy is the same as in the case of one centroid. The test file $F$ is classified to the model with the closest distance.

A third method is also tested. Here we use a set of exemplar files of each type as centroids. Thus, a set of randomly chosen normal files for each file type are used as centroids. There are $N$ models if there are $N$ chosen exemplar files. We also analyze the accuracy of the method using different truncations - first 10, 50, 100, 200, 400, 600, 1000, 2000, 4000, 6000 , and 8000 bytes, and the entire file. In this experiment, we evaluate both 1-gram and 2-gram analysis.

We trained models on $80 \%$ of the randomly selected files of each group (normal and malicious) to build a set of models for each class. The remaining $20 \%$ of the files are used as test files. Again, we know ground truth and hence can accurately evaluate performance. Note that all of the malicious files extensions are EXE. For each of the test files, we evaluate their distance from both the "normal model" and the "malicious model". 31 normal application executable files, 45 spyware, 331 normal executable under folder System 32 and 571 viruses were tested. Three "pairs" of groups of files are tested - Normal executable vs. spyware, normal application vs. spyware and normal executable vs. viruses. We report the average accuracy over 100 trials using cross validation for each of the modeling techniques.

The results are shown in figure 6. Each column represents each modeling method: one-centroid, muli-centroids and exemplar file centroids. The rows indicate the testing "pairs". In each plot, the X and Yaxis are the false positive rate and detection rate, respectively. The asterisk marks are 1-gram tests using different truncation sizess, and the circle marks represent the results of 2-gram centoids. In these plots, the truncation sizes are not arranged in order. In these two dimensional plots, the optimum performance appears closest to the upper left corner of each 
plot. That is to say, a false positive rate of 0 and a detection rate of 1 is perfect performance.

The results show relatively good performance in some case of normal executable vs. spyware and normal executable vs. virus. Because viruses and worms usually target the System 32 folder, we can reasonable well detect non-standard malicious files in that folder. Moreover, the performance results varied under different truncation sizes. Thus, we have considerable additional analysis to perform in our future work to identify appropriate file sizes (and normalization strategies) to improve detection performance. However, the plots clearly indicate that performance varies widely, which suggests the comparison method is too weak to reliably detect malicious code.

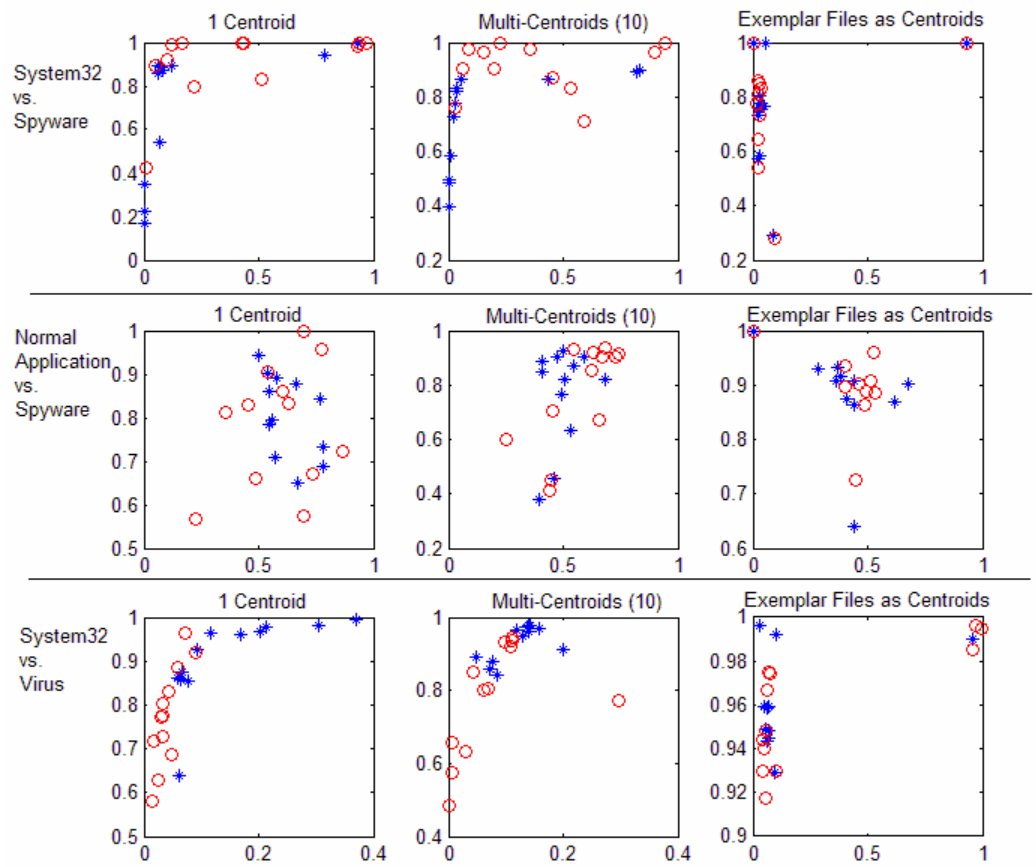

Fig. 6. 2-class classification of malware and normal EXE files. X-Axis: false positive, Y-Axis: detection rate. Asterisk marks: 1-gram test, Circle marks: 2gram test.

Notice that there is a high false positive rate in the case of testing normal applications to the Spyware samples. This is due to two reasons. First, the range of the normal application file size is too large, ranging from $10 \mathrm{~KB}$ to $10 \mathrm{MB}$. It is hard to normalize the models when the data ranges so widely. Second, the spyware files are somewhat similar to 
normal application files. They are both MS Windows applications, and they may be used for similar purposes. Hence, other features may be necessary to explore ways of better distinguishing this class of files.

In the experiments performed to date, there is no strong evidence to indicate that 2-gram analysis is better than 1-gram analysis. Even though the 1-gram memory usage is much smaller and the computation speed is much faster, we may need to analyze far more many files to determine whether the heavy price paid in performing 2-gram analysis will perform better ultimately.

\subsection{Uniform Distributions of 1-gram analysis: encrypted files and spyware}

In this experiment we scan Windows files to determine whether any are close to a uniform 1-gram distribution. We thus test whether spyware that is obfuscated by self-encryption technology may be revealed as substantially different from other executable files on a Windows host platform. We conjecture that self-encrypted files, such as stealthy Trojans and spyware, may be detectable easily via 1-gram analysis.

The normal EXE from System32, spyware and virus files used in the experiments reported in the previous section are used here again. Moreover, we randomly select 600 files (DOC, PPT, GIF, JPG, PDF, DLL) from Google, 100 for each type. Since the models are normalized, the uniform distribution is an array with uniform value $1 / n$, where $n$ is the length of the array and $n$ is 256 in the 1-gram test. For each of the test files, we compute the Manhattan distance against the uniform model and plot the distance in figure 7 . The files that are closest to uniform distribution are listed in table 7.

As the plot shows, JPG, GIF and PDF files are self-encoded, so they are more similar to the uniform distribution. System 32 files and DLL files are not self-encrypted, and most of the virus and spyware tested are also not self-encrypted. However, some of the normal files are selfencrypted and quite similar to the random distribution. An interesting example is the application ad-aware.exe, which is a COTS adware detection application that apparently uses self-encryption, perhaps to attempt to protect its intellectual property. 

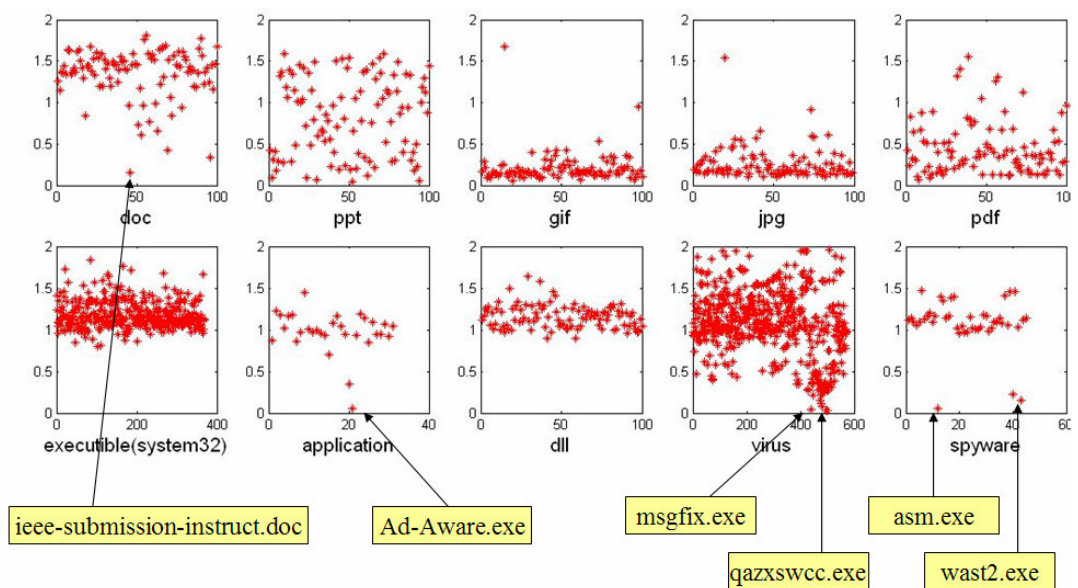

ieee-submission-instruct.doc

Ad-Aware.exe

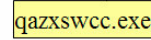

wast2.exe

Fig. 7. The distance of testing files against the uniform distribution. X-Axis: the test files, Y-Axis: the distance.

Table 7. Files whose content is deemed close to a uniform 1-gram distribution (hence likely encrypted).

\begin{tabular}{|l|l|}
\hline File name & Description \\
\hline $\begin{array}{l}\text { Ieee-submission- } \\
\text { instruct.doc }\end{array}$ & $\begin{array}{l}\text { An ieee submission format instruction Word file. It is } \\
\text { unclear why this file follows a normal distribution. }\end{array}$ \\
\hline Ad-Aware.exe & $\begin{array}{l}\text { Ad-Aware.exe: ad-aware from lavasoft, searches and } \\
\text { removes spyware and/or adware programs from your } \\
\text { computer. }\end{array}$ \\
\hline msgfix.exe & $\begin{array}{l}\text { msgfix.exe is the W32.Gaobot.SN Trojan. This Trojan } \\
\text { allows attackers to access your computer, stealing } \\
\text { passwords and personal data. }\end{array}$ \\
\hline Qazxswcc.exe & $\begin{array}{l}\text { qazxswcc.exe is as a backdoor Trojan. } \\
\text { asm.exe is a commercial spyware program by Gator. This } \\
\text { program monitors browsing habits and distributes the } \\
\text { data back to a Gator server for analysis. This also } \\
\text { prompts advertising pop-ups. }\end{array}$ \\
\hline wast2.exe & $\begin{array}{l}\text { wast2.exe is an adware based Internet Explorer browser } \\
\text { helper object that delivers targeted ads based on a user's } \\
\text { browsing patterns. }\end{array}$ \\
\hline
\end{tabular}




\section{Concluding Remarks}

In this paper, we demonstrate that simple techniques to embed known malcode in normal files can easily bypass signature-based detection. We successfully inserted known malcode in non-executable (PDF and DOC) files without being detected by AV scanners, and several were normally opened and executed. Various code obfuscation techniques can also be used by crafty attackers to avoid inspection by signature-based methods. We propose an alternative approach to augment existing signature-based protection mechanisms with statistical content analysis techniques. Rather than only scanning for signatures, we compute the statistical binary content of files in order to detect anomalous files or portions of files which may indicate a malcode embedding. Although it may be relatively easy to detect tainted files where malcode is embedded in the head (where normal meta-data is expected) or at the tail of a file, detecting embeddings within the interior portion of a file poses a significant challenge. The results show that far more work is needed to identify files tainted by stealthy malcode embeddings. On the positive side, self-encrypted files are relatively easy to spot.

The results reported here are preliminary, and have opened up other avenues of future work. For example, adherence to a 1-gram model may not be the right strategy. Higher order grams may reveal more structure in files, and help identify unusual segments worthy of deeper analysis. Furthermore, file formats are defined by, typically, proprietary and unpublished syntactic conventions providing markers delimiting regions of files handled different (eg., embedded objects with specialized methods for their processing) that may be analyzed by alternative methods. Utilizing this information may provide a finer granularity of modeling normal file formats and perhaps produce improved performance.

Finally, we believe another path may be useful, profiling application execution when opening typical/normal files. It may be possible to identify portions of files that harbor malcode by finding possible deviations from normal application behavior. Combining static analysis with dynamic program behavior analysis may be the best option for detecting tainted files with embedded stealthy malcode.

References:

[1] K. G. Anagnostakis, S. Sidiroglou, P. Akritidis, K.Xinidis, E. Markatos, and A. D. Keromytis. "Detecting Targeted Attacks Using Shadow Honeypots", Proceedings of the $14^{\text {th }}$ USENIX Security Symposium, 2005. 
[2] R. Balzer and N. Goldman, "Mediating Connectors", Proceedings of the 19 IEEE International Conference on Distributed Computing Systems Workshop, 1994.

[3] M. Christodorescu and S. Jha, "Static Analysis of Executables to Detect Malicious Patterns", In Proceedings of the 12th USENIX Security Symposium, August 2003

[4] M. Damashek. "Gauging similarity with n-grams: language independent categorization of text." Science, 267(5199):843--848, 1995

[5] E. Eskin, W. Lee and S. J. Stolfo. “Modeling System Calls for Intrusion Detection with Dynamic Window Sizes." Proceedings of DISCEX II. June 2001.

[6] J. Giffin, S. Jha, and B. Miller. "Detecting Manipulated Remote Call Streams". In the $11^{\text {th }}$ USENIX Security Symposium, 2002

[7] C. Ko, M. Ruschitzka, and K. Levitt. "Execution monitoring of securitycritical programs in distributed systems: A specification-based approach". In Proceedings of the IEEE Symposium on Security and Privacy, 1997.

[8] J. Kolter and M. Maloof. "Learning to Detect Malicious Executables in the Wild." In the Proceedings of ACM SIGKDD, 2004

[9] C. Krugel, W. Robertson, F. Valeur, G. Vigna. "Static Disassembly of Obfuscated Binaries”. In Proceedings of USENIX Security Symposium, 2004.

[10] A. Kurchuk and A. Keromytis. "Recursive Sandboxes: Extending Systrace to Empower Applications". In Proceeding of the $19^{\text {th }}$ IFIP International Information Security Conference (SEC), Aug. 2004

[11] W. Li, K. Wang, S. Stolfo and B. Herzog. "Fileprints: identifying file types by n-gram analysis". $6^{\text {th }}$ IEEE Information Assurance Workshop, West Point, NY, June, 2005.

[12] M. McDaniel, M. Heydari. "Content Based File Type Detection Algorithms". $36^{\text {th }}$ Annual Hawaii International Conference on System Sciences (HICSS'03).

[13] G. Necula, P. Lee. "Proof-Carrying Code" In Proceedings of the 24th ACM SIGPLAN-SIGACT Symposium on Principles of Programming Languages (POPL), 1997

[14] F. B. Schneider. "Enforceable security politics". Technical Report 98-1664, Cornell University, 1998

[15] M. Schultz, E. Eskin, and S. Stolfo. "Malicious Email Filter - A UNIX Mail Filter that Detects Malicious Windows Executables." In Proceedings of USENIX Annual Technical Conference - FREENIX Track. Boston, MA: June 2001.

[16] R. Sekar, A. Gupta, J. Frullo, T. Shanbhag, A. Tiwari, H. Yang and S. Zhou. "Specification-Based Anomaly Detection: a new approach for detecting network intrusions", RAID 2002

[17] A. Shamir and N. van Someren. "Playing Hide and Seek with stored keys", Financial Cryptography 1999.

[18] D. Wagner and D. Dean. "Intrusion Detection via Static Analysis". In IEEE Symposium in Security and Privacy, Oakland, CA, 2001

[19] K. Wang, G. Cretu and S. Stolfo. "Anomalous Payload-based Worm Detection and Signature Generation". To appear in Proceedings of the Eighth 
International Symposium on Recent Advances in Intrusion Detection, Sept. 2005.

[20] K. Wang and S. Stolfo. “Anomalous Payload-based Network Intrusion Detection". In Proceedings of the Seventh International Symposium on Recent Advance in Intrusion Detection (RAID), Sept. 2004.

[21] C. Warrender, S. Forrest, and B. Pearlmutter. "Detecting intrusions using System calls: alternative data models, Proc. of 1999 IEEE Symposium on Security and Privacy, 1999.

[22] VX Heavens http://vx.netlux.org/

[23] Net Alliance http://www.shellcode.com.ar/docz/bof/pe.pdf 